Bull. Korean Math. Soc. 52 (2015), No. 3, pp. 907-914

http://dx.doi.org/10.4134/BKMS.2015.52.3.907

\title{
STABILITY FOR A VISCOELASTIC PLATE EQUATION WITH $p$-LAPLACIAN
}

\author{
Sun Hye PARK
}

ABSTRACT. In this paper, we consider a viscoelastic plate equation with $p$-Laplacian

$$
u^{\prime \prime}+\Delta^{2} u-\operatorname{div}\left(|\nabla u|^{p-2} \nabla u\right)+\sigma(t) \int_{0}^{t} g(t-s) \Delta u(s) d s-\Delta u^{\prime}=0 .
$$

By introducing suitable energy and Lyapunov functionals, we establish a general decay estimate for the energy, which depends on the behavior of both $\sigma$ and $g$.

\section{Introduction}

This work is concerned with the asymptotic stability of solutions for a viscoelastic plate equation with $p$-Laplacian of the form

$$
\begin{aligned}
& u^{\prime \prime}+\Delta^{2} u-\operatorname{div}\left(|\nabla u|^{p-2} \nabla u\right) \\
& +\sigma(t) \int_{0}^{t} g(t-s) \Delta u(s) d s-\Delta u^{\prime}=0 \text { in } \Omega \times(0, \infty), \\
& u=\Delta u=0 \text { on } \Gamma \times(0, \infty), \\
& u(0)=u_{0}, u^{\prime}(0)=u_{1} \text { in } \Omega,
\end{aligned}
$$

where $\Omega$ is a bounded domain in $\mathbb{R}^{n}$ with smooth boundary $\Gamma, \sigma$ and $g$ are real functions.

This model can be regarded as a fourth order weak viscoelastic plate equation with a lower order perturbation of $p$-Laplacian type, and which is related to one-dimensional nonlinear equation of elastoplastic microstructure flows given by

$$
u^{\prime \prime}+u_{x x x x}-a\left(u_{x}^{2}\right)_{x}=0 .
$$

Received April 21, 2014; Revised August 28, 2014.

2010 Mathematics Subject Classification. 35L70, 35B40.

Key words and phrases. plate equation, $p$-laplacian, weak viscoelasticity, general decay.

This research was supported by Basic Science Research Program through the National Research Foundation of Korea(NRF) funded by the Ministry of Science, ICT and Future Planning(Grant No. 2014R1A1A3A04049561). 
As a general form of (1.4), many authors [5, 9, 11, 12] studied the following equation with appropriate boundary and initial conditions:

$$
u^{\prime \prime}+\alpha \Delta^{2} u-\operatorname{div}\left(\gamma\left(|\nabla u|^{2}\right) \nabla u\right)-\Delta u^{\prime}+h\left(u^{\prime}\right)+g(u)=f(x) .
$$

Ma and Soriano [5] proved the global existence and decay of the solutions when $\alpha=h\left(u^{\prime}\right)=0$ and $\gamma\left(|\nabla u|^{2}\right)=|\nabla u|^{p-2}$ in (1.5). Park et al. [9] improved the results in [5] by generalizing the assumptions on $h$, that is, the function $h$ is assumed to be a discontinuous and nonlinear multi-valued function. The authors in $[11,12]$ proved the global existence and asymptotic behavior of weak and strong solutions of (1.5). These works are concerned with exponential decay rates.

On the other hand, Andrade et al. [1] discussed the energy decay of solutions for problem (1.1)-(1.3) when $\sigma(t)=1$. The interaction of the memory term with $p$-Laplacian operator was first considered by them. They showed the existence of solutions and exponential decay rates of energy of solutions under the condition $g^{\prime}(t) \leq-c g(t)$ for some $c>0$. The aim of this work is to investigate the general decay of solutions for problem (1.1)-(1.3). Concerning the study of viscoelastic equations with memory, there is a substantial number of papers (see $[2,3,4,6,8,10]$ and references therein). Cavalcanti et al. [2] proved exponential and polynomial decay for the viscoelastic wave equation under the usual condition

$$
-c_{1} g(t) \leq g^{\prime}(t) \leq-c_{2} g(t) \text { and } 0 \leq g^{\prime \prime}(t) \leq c_{3} g(t)
$$

for some positive constants $c_{1}, c_{2}, c_{3}$. Since then, several authors are interested in weakening the condition of relaxation function $g$. Messaoudi and Tatar [8] introduced conditions on $g$ such as $g^{\prime}(t) \leq-\zeta g^{p}(t)$ for $1 \leq p<\frac{3}{2}$, and get the exponential and polynomial decay rates. Moreover, the authors in [4] obtained general stability for the Timoshenko system under weaker condition on $g$ such as $g^{\prime}(t) \leq-\zeta(t) g(t)$.

Recently, Messaoudi [7] considered the viscoelastic equation of the form

$$
u^{\prime \prime}-\Delta u+\sigma(t) \int_{0}^{t} g(t-s) \Delta u(s) d s=0,
$$

and proved a general decay result which depends both on the behavior of $\sigma$ and $g$. Inspired by $[1,7]$, we investigate the general decay estimate of solutions for the weak viscoelastic beam equation with $p$-Lapalcian. The results of this article extend those of [1] with respect to some aspects.

The plan of this paper is as follows. In Section 2, we give some notations and material needed for our work. In Section 3, we derive general decay of the energy of solutions for problem (1.1)-(1.3).

\section{Statement of main results}

In this section, we present some material needed in the proof of our results. Throughout this paper, $(u, v)=\int_{\Omega} u(x) v(x) d x$. For a Banach space $X,\|\cdot\|_{X}$ 
denotes the norm of $X$. For simplicity, we denote $\|\cdot\|_{L^{p}(\Omega)}$ by $\|\cdot\|_{p}$ and $\|\cdot\|_{L^{2}(\Omega)}$ by $\|\cdot\|$. Let $\lambda_{0}, \lambda_{1}$ and $\lambda_{2}$ be positive constants satisfying

$$
\lambda_{0}\|u\|^{2} \leq\|\Delta u\|^{2}, \quad \lambda_{1}\|\nabla u\|^{2} \leq\|\Delta u\|^{2}, \quad \lambda_{2}\|u\|^{2} \leq\|\nabla u\|^{2} .
$$

Now, we give assumptions on the system (1.1)-(1.3).

For $n \in \mathbb{N}$, we assume that $p$ satisfies

$$
2 \leq p \leq \frac{2 n-2}{n-2} \text { if } n \geq 3 \text { or } p \geq 2 \text { if } n=1,2 .
$$

Then, it holds

$$
H^{2}(\Omega) \cap H_{0}^{1}(\Omega) \hookrightarrow W_{0}^{1,2(p-1)}(\Omega) \hookrightarrow H_{0}^{1}(\Omega) \hookrightarrow L^{2}(\Omega) .
$$

For the relaxation function $g$ and potential $\sigma$, as in [7], we assume that $g, \sigma$ : $\mathbb{R}_{+} \rightarrow \mathbb{R}_{+}$are nonincreasing differentiable functions satisfying

$$
\begin{gathered}
g(0)>0, \quad k:=\int_{0}^{\infty} g(s) d s<\infty, \\
\sigma(t)>0, \frac{1}{2}-\frac{2 \sigma(t)}{\lambda_{1}} \int_{0}^{t} g(s) d s \geq l>0 \quad \text { for } t \geq 0,
\end{gathered}
$$

and there exist a nonincreasing differentiable function $\zeta: \mathbb{R}_{+} \rightarrow \mathbb{R}_{+}$with

$$
\zeta(t)>0, g^{\prime}(t) \leq-\zeta(t) g(t), \quad \text { for } t \geq 0, \quad \lim _{t \rightarrow \infty} \frac{-\sigma^{\prime}(t)}{\zeta(t) \sigma(t)}=0 .
$$

Theorem 2.1. If $\left(u_{0}, u_{1}\right) \in\left(H^{2}(\Omega) \cap H_{0}^{1}(\Omega)\right) \times L^{2}(\Omega)$, then problem (1.1)-(1.3) has a unique weak solution

$$
u \in C\left(0, \infty ; H^{2}(\Omega) \cap H_{0}^{1}(\Omega)\right) \cap C^{1}\left(0, \infty ; L^{2}(\Omega)\right) .
$$

Moreover, if $\left(u_{0}, u_{1}\right) \in H_{\Gamma}^{3}(\Omega) \times H_{0}^{1}(\Omega)$, where $H_{\Gamma}^{2}(\Omega)=\left\{u \in H^{3}(\Omega): u=\right.$ $\Delta u=0$ on $\Gamma\}$, then the problem (1.1)-(1.3) has a unique solution $u$ satisfying

$$
u \in L^{\infty}\left(0, \infty ; H_{\Gamma}^{3}(\Omega)\right), u^{\prime} \in L^{\infty}\left(0, T ; H_{0}^{1}(\Omega)\right), u^{\prime \prime} \in L^{2}\left(0, T ; H^{-1}(\Omega)\right) .
$$

Proof. See Andrade, Jorge Silva and Ma [1].

\section{General decay of solutions}

For simplicity of notations, we shall omit $x$ and $t$ in all functions of $x$ and $t$ if there is no ambiguity, and $c$ denotes a generic positive constant different from line to line or even in the same line, and let

$$
\begin{gathered}
g * v=\int_{0}^{t} g(t-s) v(s) d s, \\
g \square v=\int_{0}^{t} g(t-s)\|v(t)-v(s)\|^{2} d s .
\end{gathered}
$$

We introduce the energy functional $E(t)$ of the problem (1.1)-(1.3) as

$$
E(t)=\frac{1}{2}\left\|u^{\prime}(t)\right\|^{2}+\frac{1}{2}\|\Delta u(t)\|^{2}+\frac{1}{p}\|\nabla u(t)\|_{p}^{p},
$$


and a modified energy by

$$
\begin{aligned}
\mathcal{E}(t)= & \frac{1}{2}\left\|u^{\prime}(t)\right\|^{2}+\frac{1}{2}\|\Delta u(t)\|^{2}+\frac{1}{p}\|\nabla u(t)\|_{p}^{p} \\
& +\frac{\sigma(t)}{2} g \square \nabla u-\frac{\sigma(t)}{2}\left(\int_{0}^{t} g(s) d s\right)\|\nabla u(t)\|^{2} .
\end{aligned}
$$

Remark 3.1. From definitions of $E(t)$ and $\mathcal{E}(t)$, it is noted that $E(t) \leq \frac{1}{2 l} \mathcal{E}(t)$ for all $t \geq 0$. Therefore it is enough to obtain the desired decay for the modified energy $\mathcal{E}(t)$.

To demonstrate the stability of the system (1.1)-(1.3), the lemmas below are essential.

Lemma 3.1. The modified energy $\mathcal{E}(t)$ satisfies

$$
\begin{aligned}
\mathcal{E}^{\prime}(t)= & -\left\|\nabla u^{\prime}(t)\right\|^{2}+\frac{\sigma(t)}{2} g^{\prime} \square \nabla u-\frac{\sigma^{\prime}(t)}{2}\left(\int_{0}^{t} g(s) d s\right)\|\nabla u(t)\|^{2} \\
& -\frac{\sigma(t)}{2} g(t)\|\nabla u(t)\|^{2}+\frac{\sigma^{\prime}(t)}{2} g \square \nabla u .
\end{aligned}
$$

Proof. Multiplying (1.1) by $u^{\prime}(t)$, we have

$$
\frac{d}{d t} E(t)=-\left\|\nabla u^{\prime}(t)\right\|^{2}+\sigma(t)\left(g * \nabla u, \nabla u^{\prime}(t)\right) .
$$

A direct calculation gives

$$
\begin{aligned}
= & -\frac{\sigma(t)}{2} g(t)\|\nabla u\|^{2}+\frac{\sigma(t)}{2} g^{\prime} \square \nabla u-\frac{\sigma(t)}{2} \frac{d}{d t}\left(g \square \nabla u-\int_{0}^{t} g(s) d s\|\nabla u\|^{2}\right) \\
= & -\frac{d}{d t}\left(\frac{\sigma(t)}{2} g \square \nabla u-\frac{\sigma(t)}{2} \int_{0}^{t} g(s) d s\|\nabla u\|^{2}\right)-\frac{\sigma(t)}{2} g(t)\|\nabla u\|^{2} \\
& +\frac{\sigma(t)}{2} g^{\prime} \square \nabla u+\frac{\sigma^{\prime}(t)}{2} g \square \nabla u-\frac{\sigma^{\prime}(t)}{2} \int_{0}^{t} g(s) d s\|\nabla u\|^{2} .
\end{aligned}
$$

Applying this to the second term in the right hand side of (3.3), the proof is completed.

Remark 3.2. Since $-\frac{\sigma^{\prime}(t)}{2}\left(\int_{0}^{t} g(s) d s\right)\|\nabla u(t)\|^{2} \geq 0, \mathcal{E}(t)$ may not be nonincreasing.

Now, let us define the perturbed modified energy by

$$
L(t)=N \mathcal{E}(t)+\sigma(t) \Psi(t),
$$

where $\Psi(t)=\left(u^{\prime}(t), u(t)\right)$ and $N>0$.

Lemma 3.2. There exist $c_{1}>0$ and $c_{2}>0$ such that for appropriately large $N>0$

$$
c_{1} \mathcal{E}(t) \leq L(t) \leq c_{2} \mathcal{E}(t) \text { for all } t \geq 0
$$


Proof. Young's inequality gives that

$$
|\Psi(t)| \leq \frac{1}{2}\left(\left\|u^{\prime}\right\|^{2}+\|u\|^{2}\right) \leq c\left(\left\|u^{\prime}\right\|^{2}+\|\Delta u\|^{2}\right) \leq c \mathcal{E}(t) .
$$

Since $\sigma$ is non-increasing, we get

$$
|L(t)-N \mathcal{E}(t)| \leq \sigma(t)|\Psi(t)| \leq c \sigma(0) \mathcal{E}(t) .
$$

Choosing suitably $N>0$, we complete the proof.

Lemma 3.3. There exist positive constants $c_{3}, c_{4}$ and $t_{0}$ satisfying

$$
\frac{d}{d t} L(t) \leq-c_{3} \sigma(t) \mathcal{E}(t)+c_{4} \sigma(t) g \square \nabla u \text { for } t \geq t_{0} .
$$

Proof. Using the problem (1.1), we have

$$
\Psi^{\prime}(t)=\left\|u^{\prime}\right\|^{2}-\|\Delta u\|^{2}-\|\nabla u\|_{p}^{p}+\sigma(t)(g * \nabla u, \nabla u)-\left(\nabla u, \nabla u^{\prime}\right) .
$$

Young's inequality and (2.1) give that

$$
\begin{aligned}
(g * \nabla u, \nabla u) & =\int_{0}^{t} g(t-s)(\nabla u(s)-\nabla u(t), \nabla u(t)) d s+\int_{0}^{t} g(s) d s\|\nabla u(t)\|^{2} \\
& \leq 2 \int_{0}^{t} g(s) d s\|\nabla u\|^{2}+\frac{1}{4} g \square \nabla u \\
& \leq \frac{2 \sigma(t)}{\lambda_{1}} \int_{0}^{t} g(s) d s\|\Delta u\|^{2}+\frac{1}{4} g \square \nabla u
\end{aligned}
$$

and

$$
-\left(\nabla u, \nabla u^{\prime}\right) \leq \frac{1}{2}\|\Delta u\|^{2}+\frac{1}{2 \lambda_{1}}\left\|\nabla u^{\prime}\right\|^{2} .
$$

Thus, we obtain

$$
\begin{aligned}
\Psi^{\prime}(t) \leq & \left(\frac{1}{\lambda_{2}}+\frac{1}{2 \lambda_{1}}\right)\left\|\nabla u^{\prime}(t)\right\|^{2}-\left(\frac{1}{2}-\frac{2 \sigma(t)}{\lambda_{1}} \int_{0}^{t} g(s) d s\right)\|\Delta u(t)\|^{2} \\
& -\|\nabla u(t)\|_{p}^{p}+\frac{\sigma(t)}{4} g \square \nabla u .
\end{aligned}
$$

Combining (3.2), (3.5) and (3.7), we have

$$
\begin{aligned}
\frac{d}{d t} L(t)= & N \mathcal{E}^{\prime}(t)+\sigma(t) \Psi^{\prime}(t)+\sigma^{\prime}(t) \Psi(t) \\
\leq & -N\left\|\nabla u^{\prime}\right\|^{2}-\frac{N \sigma^{\prime}(t)}{2} \int_{0}^{t} g(s) d s\|\nabla u\|^{2}+\sigma(t)\left(\frac{1}{\lambda_{2}}+\frac{1}{2 \lambda_{1}}\right)\left\|\nabla u^{\prime}\right\|^{2} \\
& -\sigma(t)\left(\frac{1}{2}-\frac{2 \sigma(t)}{\lambda_{1}} \int_{0}^{t} g(s) d s\right)\|\Delta u\|^{2}-\sigma(t)\|\nabla u\|_{p}^{p} \\
& +\frac{\sigma^{2}(t)}{4} g \square \nabla u+\sigma^{\prime}(t)\left(u, u^{\prime}\right) .
\end{aligned}
$$


Using

$$
\sigma^{\prime}(t)\left(u, u^{\prime}\right) \leq-\frac{\sigma^{\prime}(t)}{2 \lambda_{0}}\|\Delta u\|^{2}-\frac{\sigma^{\prime}(t)}{2 \lambda_{2}}\left\|\nabla u^{\prime}\right\|^{2}
$$

we get

$$
\begin{aligned}
& \frac{d}{d t} L(t) \\
\leq & -\left(N-\sigma(t)\left(\frac{1}{\lambda_{2}}+\frac{1}{2 \lambda_{1}}\right)+\frac{\sigma^{\prime}(t)}{2 \lambda_{2}}\right)\left\|\nabla u^{\prime}\right\|^{2}-\sigma(t)\|\nabla u\|_{p}^{p}+\frac{\sigma^{2}(t)}{4} g \square \nabla u \\
& -\left(\sigma(t)\left(\frac{1}{2}-\frac{2 \sigma(t)}{\lambda_{1}} \int_{0}^{t} g(s) d s\right)+\frac{N \sigma^{\prime}(t)}{2 \lambda_{1}} \int_{0}^{t} g(s) d s+\frac{\sigma^{\prime}(t)}{2 \lambda_{0}}\right)\|\Delta u\|^{2} .
\end{aligned}
$$

Noting that (2.3) and the relations

$$
\frac{\sigma(t)}{\sigma(0)} \leq 1 \text { and } \int_{0}^{t} g(s) d s \leq k,
$$

we arrive

$$
\begin{aligned}
\frac{d}{d t} L(t) \leq & -\sigma(t)\left(\frac{N}{\sigma(0)}-\left(\frac{1}{\lambda_{2}}+\frac{1}{2 \lambda_{1}}\right)+\frac{\sigma^{\prime}(t)}{2 \lambda_{2} \sigma(t)}\right)\left\|\nabla u^{\prime}\right\|^{2}-\sigma(t)\|\nabla u\|_{p}^{p} \\
& +\frac{\sigma^{2}(t)}{4} g \square \nabla u-\sigma(t)\left(l+\frac{N k \sigma^{\prime}(t)}{2 \lambda_{1} \sigma(t)}+\frac{\sigma^{\prime}(t)}{2 \lambda_{0} \sigma(t)}\right)\|\Delta u\|^{2} .
\end{aligned}
$$

First, we take $N>0$ such that

$$
\frac{N}{\sigma(0)}-\left(\frac{1}{\lambda_{2}}+\frac{1}{2 \lambda_{1}}\right)>0 .
$$

Since

$$
\lim _{t \rightarrow \infty} \frac{-\sigma^{\prime}(t)}{\sigma(t)}=0
$$

we can choose $t_{0}>0$ sufficiently large so that, for $t \geq t_{0}$,

$$
\frac{N}{\sigma(0)}-\left(\frac{1}{\lambda_{2}}+\frac{1}{2 \lambda_{1}}\right)+\frac{\sigma^{\prime}(t)}{2 \lambda_{2} \sigma(t)}>0
$$

and

$$
l+\frac{N k \sigma^{\prime}(t)}{2 \lambda_{1} \sigma(t)}+\frac{\sigma^{\prime}(t)}{2 \lambda_{0} \sigma(t)}>0 .
$$

This completes the proof.

Our main theorem is the following.

Theorem 3.1. There exist positive constants $C_{0}, \omega$ and $t_{1}$ such that

$$
E(t) \leq C_{0} e^{-\omega \int_{0}^{t} \sigma(s) \zeta(s) d s} \quad \text { for } t \geq t_{1} .
$$


Proof. Multiplying (3.6) by $\zeta(t)$, using (2.4) and (3.2), we get

$$
\begin{aligned}
\zeta(t) L^{\prime}(t) & \leq-c_{3} \sigma(t) \zeta(t) \mathcal{E}(t)+c_{4} \sigma(t) \zeta(t) g \square \nabla u \\
& \leq-c_{3} \sigma(t) \zeta(t) \mathcal{E}(t)-c_{4} \sigma(t) g^{\prime} \square \nabla u \\
& \leq-c_{3} \sigma(t) \zeta(t) \mathcal{E}(t)+c_{4}\left(-2 \mathcal{E}^{\prime}(t)-\sigma^{\prime}(t) \int_{0}^{t} g(s) d s\|\nabla u(t)\|^{2}\right) .
\end{aligned}
$$

Since $\zeta$ is nonincreasing, by (2.1) and (2.2), one sees that

$$
\begin{aligned}
\left(\zeta(t) L(t)+2 c_{4} \mathcal{E}(t)\right)^{\prime} & \leq-c_{3} \sigma(t) \zeta(t) \mathcal{E}(t)-\frac{c_{4} \sigma^{\prime}(t)}{\lambda_{1}} \int_{0}^{t} g(s) d s\|\Delta u(t)\|^{2} \\
& \leq-\sigma(t) \zeta(t)\left(c_{3}+\frac{c_{4} k \sigma^{\prime}(t)}{\lambda_{1} l \sigma(t) \zeta(t)}\right) \mathcal{E}(t) \quad \text { for } t \geq t_{0}
\end{aligned}
$$

Since $\lim _{t \rightarrow \infty} \frac{-\sigma^{\prime}(t)}{\zeta(t) \sigma(t)}=0$, we can choose $t_{1} \geq t_{0}$ such that

$$
\left(\zeta(t) L(t)+2 c_{4} \mathcal{E}(t)\right)^{\prime} \leq-\frac{c_{3} \sigma(t) \zeta(t)}{2} \mathcal{E}(t) \text { for } t \geq t_{1} .
$$

Thus, by letting $\mathcal{L}(t)=\zeta(t) L(t)+2 c_{4} \mathcal{E}(t)$ and $c_{5}=\frac{c_{3}}{2}$, we get

$$
\mathcal{L}^{\prime}(t) \leq-c_{5} \sigma(t) \zeta(t) \mathcal{E}(t) \text { for } t \geq t_{1} .
$$

Since $\zeta(t)$ is a nonincreasing positive function, we can easily observe that $\mathcal{L}(t)$ is equivalent to $\mathcal{E}(t)$. Subsequently, it follows that

$$
\mathcal{L}^{\prime}(t) \leq-c \sigma(t) \zeta(t) \mathcal{L}(t) \text { for } t \geq t_{1}
$$

Integrating this over $\left(t_{1}, t\right)$, we conclude that

$$
\mathcal{L}(t) \leq \mathcal{L}\left(t_{1}\right) e^{-c \int_{t_{1}}^{t} \sigma(s) \zeta(s) d s} \quad \text { for all } t \geq t_{1} .
$$

The equivalent relations of $\mathcal{L}, L$, and $\mathcal{E}$ yield

$$
E(t) \leq c_{6} e^{-c \int_{t_{1}}^{t} \sigma(s) \zeta(s) d s} \text { for all } t \geq t_{1},
$$

for some positive constant $c_{6}$. By the virtue of the continuity and boundedness of $E(t)$ in the interval $\left[0, t_{1}\right]$, we complete the proof.

\section{References}

[1] D. Andrade, M. A. Jorge Silva, and T. F. Ma, Exponential stability for a plate equation with p-Laplacian and memory terms, Math. Methods Appl. Sci. 35 (2012), no. 4, 417426.

[2] M. M. Cavalcanti, V. N. Domingos Cavalcanti, J. S. Prates Filho, and J. A. Soriano, Existence and uniform decay rates for viscoelastic problems with nonlinear boundary damping, Differential Integral Equations 14 (2001), no. 1, 85-116.

[3] M. M. Cavalcanti, V. N. Domingos Cavalcanti, and P. Martinez, General decay rate estimates for viscoelastic dissipative systems, Nonlinear Anal. 68 (2008), no. 1, 177193.

[4] A. Guesmia and S. A. Messaoudi, General energy decay estimates of Timoshenko systems with frictional versus viscoelastic damping, Math. Methods Appl. Sci. 32 (2009), no. 16, 2102-2122. 
[5] T. F. Ma and J. A. Soriano, On weak solutions for an evolution equation with exponential nonlinearities, Nonlinear Anal. 37 (1999), no. 8, 1029-1038.

[6] S. A. Messaoudi, General decay of solutions of a viscoelastic equation, J. Math. Anal. Appl. 341 (2008), no. 2, 1457-1467.

[7] General decay of solutions of a weak viscoelastic equation, Arab. J. Sci. Eng. 36 (2011), no. 8, 1569-1579.

[8] S. A. Messaoudi and N. E. Tatar, Exponential and polynomial decay for a quasilinear viscoelastic equation, Nonlinear Anal. 68 (2008), no. 4, 785-793.

[9] J. Y. Park, H. M. Kim, and S. H. Park, On weak solutions for hyperbolic differential inclusion with discontinuous nonlinearities, Nonlinear Anal. 55 (2003), no. 1-2, 103-113.

[10] J. Y. Park and S. H. Park, General decay for quasilinear viscoelastic equations with nonlinear weak damping, J. Math. Phys. 50 (2009), no. 8, 10pp.

[11] Z. Yang, Longtime behavior for a nonlinear wave equation arising in elasto-plastic flow, Math. Methods Appl. Sci. 32 (2009), no. 9, 1082-1104.

[12] Z. Yang and B. Jin, Global attractor for a class of Kirchhoff models, J. Math. Phys. 50 (2009), no. 3, 29pp.

Center for Education Accreditation

Pusan National University

BUSAN 609-735, Korea

E-mail address: sh-park@pusan.ac.kr 